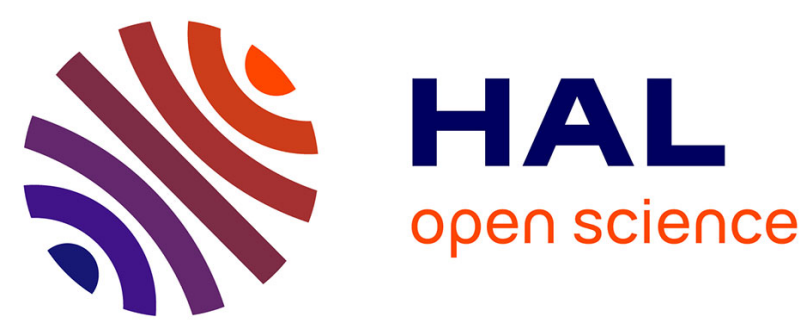

\title{
Primary crossflow vortices, secondary absolute instabilities and their control in the rotating-disk boundary layer \\ Benoît Pier
}

\section{- To cite this version:}

Benoît Pier. Primary crossflow vortices, secondary absolute instabilities and their control in the rotating-disk boundary layer. Journal of Engineering Mathematics, 2007, 57, pp.237-251. 10.1007/s10665-006-9095-5 . hal-00197478

\section{HAL Id: hal-00197478 \\ https://hal.science/hal-00197478}

Submitted on 14 Dec 2007

HAL is a multi-disciplinary open access archive for the deposit and dissemination of scientific research documents, whether they are published or not. The documents may come from teaching and research institutions in France or abroad, or from public or private research centers.
L'archive ouverte pluridisciplinaire HAL, est destinée au dépôt et à la diffusion de documents scientifiques de niveau recherche, publiés ou non, émanant des établissements d'enseignement et de recherche français ou étrangers, des laboratoires publics ou privés. 


\title{
Primary crossflow vortices, secondary absolute instabilities and their control in the rotating-disk boundary layer
}

\author{
Benoît PIER \\ Laboratoire de mécanique des fluides et d'acoustique \\ École centrale de Lyon - CNRS - Université Claude-Bernard Lyon I - INSA \\ 36 avenue Guy-de-Collongue \\ 69134 Écully cedex, France
}

$29 / 8 / 2006$

\begin{abstract}
The three-dimensional boundary layer produced by a disk rotating in otherwise still fluid is analytically investigated and its stability properties are systematically established. Using a local parallel flow approximation, finite-amplitude primary travelling vortices governed by a nonlinear dispersion relation are obtained. A secondary stability analysis yields the secondary linear dispersion relation and the secondary absolute growth rate, which determines the long term stability of the primary nonlinear vortex-trains. By using these local characteristics, spatially developing global patterns of crossflow vortices are derived by employing asymptotic techniques. This approach accounts for both the self-sustained behaviour, exhibiting a sharp transition from laminar to turbulent flow, and the spatial response to external harmonic forcing, for which onset of nonlinearity and transition both depend on the forcing parameters. Based on these results, an open-loop control method is described in detail. Its aim is not to suppress the primary fluctuations but rather to enhance them and to tune them to externally imposed frequency and modenumber, and thereby to delay onset of secondary absolute instability and transition. It is shown that transition can be delayed by more than 100 boundary layer units.
\end{abstract}

Keywords: absolute instabilities, boundary layers, control, rotating disk

\section{Introduction}

The von Kármán [37] boundary layer on an infinite disk rotating in otherwise still fluid is certainly a rather crude and academic representation of centrifugal pumps, fans, turbomachinery elements, or backwards swept aircraft wings. However, despite its simplicity, it displays most of the features observed in situations of higher complexity or with more elaborate geometries. All these types of boundary layers display similar three-dimensional velocity profiles, are subject to inviscid crossflow instabilities and rapidly undergo transition to turbulent flow [34, 36, 35]. Thus, ever since the pioneering work of von Kármán [37] and Gregory, Stuart and Walker [13], the rotating disk flow has served as the archetypal three-dimensional boundary layer, and its study has lead to many results of considerable practical importance, e.g., to the aeronautics industry.

(C) 2006 Kluwer Academic Publishers. Printed in the Netherlands. 
In the rotating-disk flow, the magnitude of the local boundary layer velocity profiles increases linearly with radial distance. As a result, the nature of the local stability features successively displays the three well-known régimes from the disk axis outwards: stability, convective instability, absolute instability (for theoretical definitions see $[16,15]$ ). Lingwood's theoretical local linear stability analyses [19] have revealed that absolute instability first occurs at a critical radius closely corresponding to the experimentally observed transition from laminar to turbulent flow. Her findings suggested that the onset of absolute instability is the driving mechanism responsible for the self-sustained time-dependent flow. Indeed, in stable or convectively unstable systems, perturbations either decay or are carried away by basic advection so that, at given position, the flow returns to its unpertubed state in the long term. It is only when the instability is absolute that an initial disturbance may grow in time at fixed spatial position and thus lead to a permanently perturbed flow. While this scenario for the behaviour fo the rotating-disk flow seems to be confirmed by most experimental studies (including Lingwood's [20]), it does not however take into account two major effects: spatial inhomogeneity and nonlinearity.

Global stability analyses of spatially inhomogenous systems governed by strictly linear dynamics are by now fairly complete. For the linear complex Ginzburg-Landau equation with spatially varying coefficients, Chomaz et al. [5] demonstrated that the complex frequency of a linear global mode is determined by a saddle point condition applied to the local linear dispersion relation. According to Monkewitz et al. [22], the same criterion also holds for the Navier-Stokes equations linearized about an arbitrary slowly varying basic flow. In this linear setting, local absolute instability is a necessary but not sufficient condition for global instability: in general, the existence of unstable global modes requires a finite range of local absolute instability. The question whether or not the local absolute instability in the rotating-disk boundary layer is strong enough to lead to unstable linear global modes has recently been addressed via direct numerical simulations [9] (see also [10] in this volume). These simulations have shown that the local absolute instability of this flow does not produce a linear amplified global mode and is only associated with a transient temporal growth; a result also in agreement with analytical developments $[12,24]$. These findings seem to be further supported by recent experimental work [23], carefully designed to remain within the linear régime.

Thus it appears that the self-sustained transition experimentally observed in the rotating-disk flow cannot be explained within linear hydrodynamic stability theory applied to spatially developing flows: a fully nonlinear approach is thus required. The study of finite-amplitude 
states covering spatially inhomogenous systems [6, 29, 7, 33, 8, 32, 30] has shown that there exists a variety of nonlinear global modes. The main result [32] of interest here is that now local absolute instability is a necessary and sufficient condition: nonlinear global modes exist however small the absolutely unstable domain. In this context, nonlinear global modes may exist in globally linearly stable media and their onset occurs via a saddle-node bifurcation [32].

Thus Lingwood's scenario can be restored by taking into account both spatial inhomogeneity and nonlinearity: the rotating-disk boundary layer is locally absolutely unstable [19], globally linearly stable [9] and globally nonlinearly unstable [27].

The discrepancy between the global linear and nonlinear dynamics is, among others, due to the important radial outflow and the large convectively unstable region upstream of the absolutely unstable region. Thus even small external perturbations may undergo a strong transient amplification and trigger nonlinear dynamics. When external perturbations are switched off, such an externally forced perturbed state would decay in the long term according to linear theory, but can survive forever due to nonlinear interactions if finite amplitudes are reached. Most experimental studies [13, 18, 38, 17] have focused on perturbations that are fixed with respect to the disk, generated by roughness elements and permanently applied. Two already mentioned studies $[20,23]$ specifically address the impulse response and the related issue of self-sustained disturbances. In these two experiments, a short air pulse is applied either through a hole in the disk surface once every disk rotation [20] or from above the boundary layer at independent timings [23]. So far the competition between self-sustained and externally forced dynamics has not been investigated in terms of complete hydrodynamic linear and nonlinear stability analyses.

The present investigation outlines a new control method where a carefully-designed periodic forcing is continuously applied in the convectively unstable region so as to modify the self-sustained nonlinear dynamics and to delay onset of transition.

This contribution first reviews and extends recent results from [25, $26,27,28]$, and then applies them to control the flow and delay transition beyond a radius at which the unforced flow would have become transitional. By systematically computing primary (Sect. 3) and secondary (Sect. 4) stability characteristics, and using asymptotic developments (Sect. 5), the naturally selected flow dynamics is explained (Sect. 6) and the spatial response to localized harmonic forcing established (Sect. 7). Based on these results, a new open-loop control method to delay transition is described (Sect. 8).

cdisk.tex; 29/8/2006; ; p.3 


\section{Basic flow structure}

The infinite disk problem lacks a characteristic length scale and thus allows the use of non-dimensional variables based on disk rotation rate, fluid viscosity and density, so that the flow does not depend on any control parameter. Throughout this paper an inertial frame of reference is used with $r, \theta$ and $z$ denoting radial, azimuthal and axial coordinates respectively. The time-independent axisymmetric basic

flow is then given by von Kármán's [37] exact similarity solution to the Navier-Stokes equations as

$$
\mathbf{U}(r, z) \equiv\left(\begin{array}{c}
r U(z) \\
r V(z) \\
W(z)
\end{array}\right) \quad \text { and } \quad P(z)
$$

where $r U, r V$ and $W$ are the non-dimensional radial, azimuthal and axial velocity components, and $P$ is the pressure.

The boundary layer thickness is constant, of order unity in nondimensional coordinates. As a result, when investigating features far from the disk axis and near a given radial location $R \gg 1$, the assumption of slow radial development is appropriate and local properties at given $r=R$ are derived by freezing the radial dependence of the basic flow (1) and studying the corresponding homogenous three-dimensional flow $\mathbf{U}(z ; R) \equiv(R U(z), R V(z), W(z))$. The value of $R$ then appears as a control parameter rather than a coordinate and plays the rôle of an effective local Reynolds number.

\section{Local linear and nonlinear travelling vortices}

In subsequent developments, the total instantaneous flow field prevailing at a given location $R$ is separated into basic and perturbation quantities according to

$$
\begin{cases}\mathbf{U}(z ; R) & +\mathbf{u}(r, \theta, z, t) \\ P(z) & +p(r, \theta, z, t)\end{cases}
$$

Local linear instability properties are then derived by assuming infinitesimally small velocity and pressure disturbances, written in normalmode form as

$$
\left\{\begin{array}{l}
\mathbf{u}(r, \theta, z, t)=\mathbf{u}^{l}(z ; \alpha, \beta ; R) \exp \mathrm{i}(\alpha r+\beta \theta-\omega t), \\
p(r, \theta, z, t)=p^{l}(z ; \alpha, \beta ; R) \exp \mathrm{i}(\alpha r+\beta \theta-\omega t),
\end{array}\right.
$$

where $\alpha$ is a complex radial wavenumber, $\beta$ an integer azimuthal modenumber, $\omega$ a complex angular frequency and $\mathbf{u}^{l}, p^{l}$ the associated complex velocity and pressure components. Substitution of (3) into the 
linearized version of the local governing equations yields an eigenvalue problem in the $z$-direction. From it, the local linear dispersion relation

$$
\omega=\Omega^{l}(\alpha, \beta ; R),
$$

together with the eigenfunctions $\mathbf{u}^{l}(z ; \alpha, \beta ; R)$ and $p^{l}(z ; \alpha, \beta ; R)$ are routinely derived. Complex values of $\Omega^{l}$ computed in the $(\alpha, \beta)$-plane for $R=450$ and $R=550$ are shown in figure 1(a1,a2,b1,b2).

In the context of open flows $[16,15]$, a crucial feature is the complex absolute frequency $\omega_{0}$ and the associated absolute wavenumber $\alpha_{0}$, defined by a vanishing group velocity condition $[4,2]$ as

$$
\omega_{0}(\beta ; R)=\Omega^{l}\left(\alpha_{0}, \beta ; R\right) \quad \text { with } \quad \frac{\partial \Omega^{l}}{\partial \alpha}\left(\alpha_{0}, \beta ; R\right)=0 .
$$

The linear instability properties of the rotating-disk boundary layer are well known [19, 21]. Local growth rates increase with radial distance away from the disk axis: the central region $R<R^{s c} \simeq 285$ is linearly stable, convective instability prevails for $R^{s c}<R<R^{c a} \simeq 507$, and absolute instability in the outer region $R>R^{c a}$. Transition from the convectively unstable (CU) to the absolutely unstable (AU) domains occurs at $R^{c a}$ for an azimuthal modenumber of $\beta^{c a}$ and with a marginal real absolute frequency of $\omega_{0}^{c a}$, where

$$
\omega_{0}^{c a} \simeq 50.5, \quad \beta^{c a}=68, \quad R^{c a} \simeq 507 .
$$

In regions of linear instability, the three-dimensional boundary layer admits nonlinearly saturated travelling crossflow vortices, governed by the complete nonlinear equations. The finite-amplitude perturbation velocity and pressure fields of these nonlinear wave solutions are of the form

$$
\left\{\begin{array}{l}
\mathbf{u}(r, \theta, z, t)=\mathbf{u}^{n l}(z, \alpha r+\beta \theta-\omega t ; \alpha, \beta ; R), \\
p(r, \theta, z, t)=p^{n l}(z, \alpha r+\beta \theta-\omega t ; \alpha, \beta ; R),
\end{array}\right.
$$

where the functions $\mathbf{u}^{n l}$ and $p^{n l}$ are $2 \pi$-periodic in their second variable $\phi \equiv \alpha r+\beta \theta-\omega t$ with $\alpha$ and $\omega$ now real quantities. After expanding $\mathbf{u}^{n l}$ and $p^{n l}$ as Fourier series in $\phi$ and substituting them into the local nonlinear governing equations, these finite-amplitude spiral waves are numerically obtained by a Newton-Raphson search procedure [26]. The real frequency $\omega$ of the saturated waves is then determined by the local nonlinear dispersion relation

$$
\omega=\Omega^{n l}(\alpha, \beta ; R)
$$

Values of $\Omega^{n l}$, computed in the domain of the $(\alpha, \beta)$-plane where nonlinear travelling vortices exist and delimited by $\Omega_{i}^{l}=0$, are shown in figure 1 (a3) and (b3) for $R=450$ and $R=550$ respectively. 

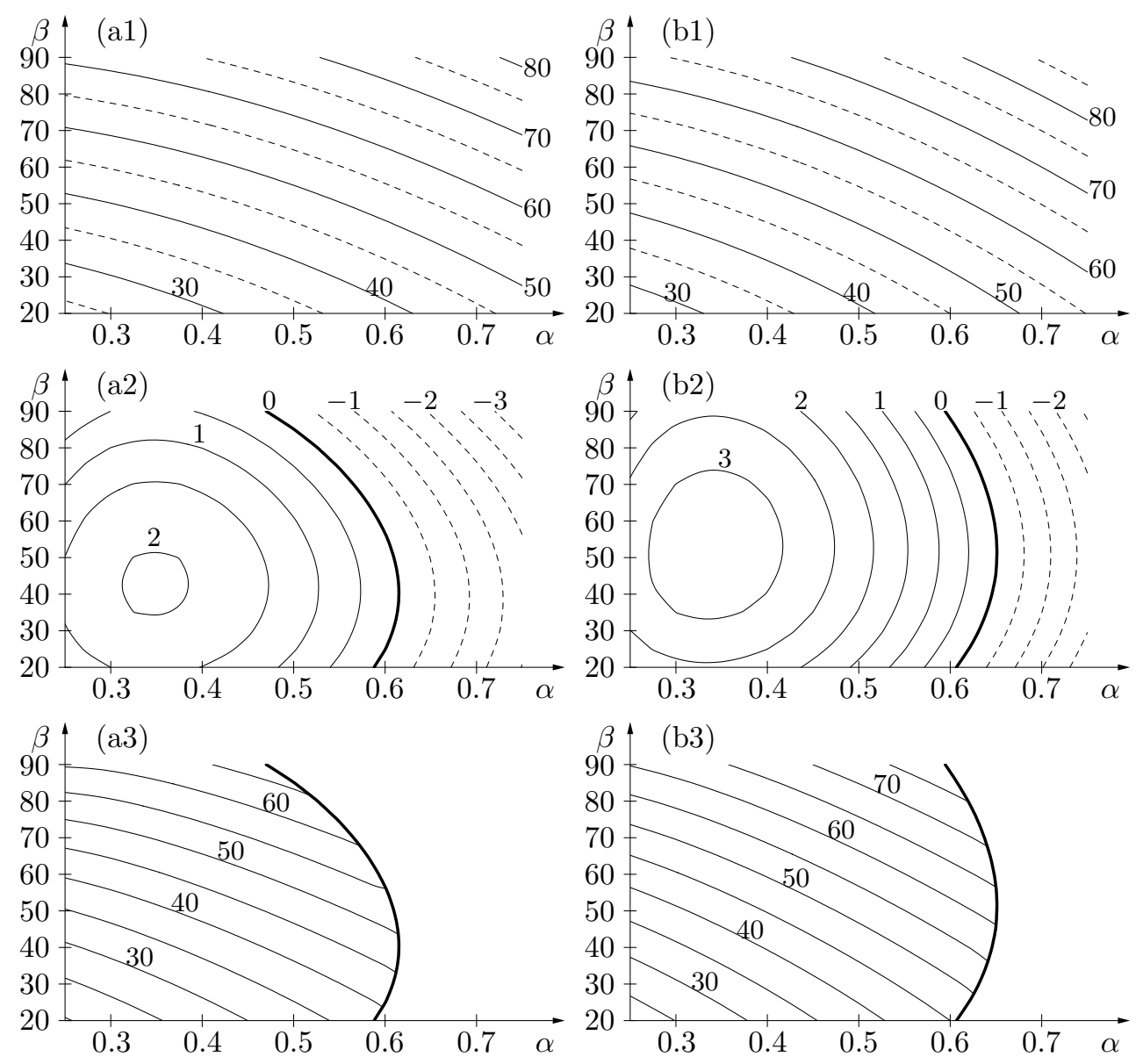

Figure 1. Local linear and nonlinear dispersion relations in $(\alpha, \beta)$-plane for (a) $R=450$ and (b) $R=550$. (a1,b1) Isocontours of linear real frequency $\Omega_{r}^{l}$. $(\mathrm{a} 2, \mathrm{~b} 2)$ Isocontours of linear temporal growth rate $\Omega_{i}^{l}$. (a3,b3) Isocontours of nonlinear frequency $\Omega^{n l}$, in subregion of $(\alpha, \beta)$-plane where saturated wave solutions exist.

\section{Secondary stability analyses}

In order to investigate the stability of the above primary finite-amplitude crossflow vortices (7) with respect to secondary perturbations, a secondary stability analysis needs to be carried out. For nonlinear travelling waves of wavenumber $\alpha$, modenumber $\beta$ and frequency $\omega$ at a radial station $R$, the total flow fields are then decomposed as

$$
\left\{\begin{array}{l}
\mathbf{U}(z ; R)+\mathbf{u}^{n l}(z, \alpha r+\beta \theta-\omega t ; \alpha, \beta ; R)+\hat{\mathbf{u}}(r, \theta, z, t) \\
P(z)+p^{n l}(z, \alpha r+\beta \theta-\omega t ; \alpha, \beta ; R)+\hat{p}(r, \theta, z, t)
\end{array}\right.
$$


where $\mathbf{U}+\mathbf{u}^{n l}, P+p^{n l}$ represent the new basic flow solution which is perturbed by $\hat{\mathbf{u}}, \hat{p}$. Assuming infinitesimally small secondary velocity and pressure disturbances and using Floquet theory [14], the perturbation quantities are written in normal-mode form as

$$
\left\{\begin{array}{l}
\hat{\mathbf{u}}(r, \theta, z, t)=\hat{\mathbf{u}}^{l}(z, \alpha r+\beta \theta-\omega t ; \hat{\alpha}, \hat{\beta} ; \alpha, \beta ; R) \exp \mathrm{i}(\hat{\alpha} r+\hat{\beta} \theta-\hat{\omega} t), \\
\hat{p}(r, \theta, z, t)=\hat{p}^{l}(z, \alpha r+\beta \theta-\omega t ; \hat{\alpha}, \hat{\beta} ; \alpha, \beta ; R) \exp \mathrm{i}(\hat{\alpha} r+\hat{\beta} \theta-\hat{\omega} t),
\end{array}\right.
$$

where $\hat{\alpha}$ is the secondary complex radial wavenumber, $\hat{\beta}$ is the secondary integer azimuthal modenumber and $\hat{\omega}$ is the associated complex secondary frequency. The eigenfunctions $\hat{\mathbf{u}}^{l}$ and $\hat{p}^{l}$ have the same periodicity as the primary wave, i.e., are $2 \pi$-periodic in the real phase variable $\phi \equiv \alpha r+\beta \theta-\omega t$, so that a Fourier series in $\phi$ is again appropriate. Two-dimensional eigenproblems in the variables $z$ and $\phi$ are then obtained after substitution of (9) with (10) into the local governing equations and linearization about the new basic flow (see [26] for numerical details). For each primary nonlinear wave characterized by the real parameters $\alpha, \beta$ and $R$ and for each combination of $\hat{\alpha}$ and $\hat{\beta}$, the solution of the corresponding eigenproblem yields the secondary linear dispersion relation

$$
\hat{\omega}=\hat{\Omega}^{l}(\hat{\alpha}, \hat{\beta} ; \alpha, \beta ; R)
$$

together with the associated eigenfunctions $\hat{\mathbf{u}}^{l}$ and $\hat{p}^{l}$.

Whether or not the primary finite-amplitude waves are permanently affected by a secondary disturbance depends on the absolute or convective nature of the secondary instability. Indeed, in the case of secondary convective instability an external impulse only triggers a transient perturbation that is eventually carried away radially outwards, while, for secondary absolute instability, perturbations are exponentially amplified at fixed radial position.

Following Brevdo \& Bridges [3], the secondary absolute frequency $\hat{\omega}_{0}$ and absolute radial wavenumber $\hat{\alpha}_{0}$ for periodic wave solutions are obtained by a saddle point condition in the complex $\hat{\alpha}$-plane

$$
\hat{\omega}_{0}(\hat{\beta} ; \alpha, \beta ; R)=\hat{\Omega}^{l}\left(\hat{\alpha}_{0}, \hat{\beta} ; \alpha, \beta ; R\right) \quad \text { where } \quad \frac{\partial \hat{\Omega}^{l}}{\partial \hat{\alpha}}\left(\hat{\alpha}_{0}, \hat{\beta} ; \alpha, \beta ; R\right)=0
$$

which is formally analogous to the criterion of Briggs [4] and Bers [2] established for spatially homogenous systems.

The stability of a system of periodic nonlinear crossflow vortices corresponding to given values $\alpha, \beta$ and $R$ depends on the maximum secondary absolute growth rate

$$
\hat{\omega}_{0, i}^{\max }(\alpha, \beta ; R) \equiv \max _{\hat{\beta}} \operatorname{Im} \hat{\omega}_{0}(\hat{\beta} ; \alpha, \beta ; R) .
$$


Thus it is the sign of this quantity which determines whether the primary spiral vortices are stable $\left(\hat{\omega}_{0, i}^{\max }<0\right)$ or not $\left(\hat{\omega}_{0, i}^{\max }>0\right)$ in the long term with respect to secondary perturbations.

Figure 2 shows isocontours (thin solid curves) of $\hat{\omega}_{0, i}^{\max } \geq 0$ computed in the $(\alpha, \beta)$-plane for $R=500$ and $R=550$. Nonlinear crossflow vortices exist in the region delimited by the (thick solid) marginal curve $\Omega_{i}^{l}(\alpha, \beta ; R)=0$, and their nonlinear frequencies $\omega=\Omega^{n l}(\alpha, \beta ; R)$ are indicated by dashed isolines. The symbols in these plots correspond to crossflow vortices at particular values of $\beta$ and $\omega$ and will be used in Sect. 8.

\section{Spatially developing pattern of crossflow vortices}

The analyses described in the previous sections entirely characterize the local properties of the rotating-disk boundary layer: primary linear stability, primary nonlinear saturated waves, secondary stability of these finite-amplitude periodic vortices. Based on these results, we are now in a position to derive a global structure of spiral vortices developing over an extended radial domain and to express them in the form of wavetrains that are slowly modulated in the radial direction. This approach is set on a firm theoretical basis by using WKBJ asymptotic techniques [1, Chap. 10].

In the rotating disk flow, the region of particular interest is the neighbourhood of $R^{c a} \simeq 507$ where onset of primary absolute instability first occurs. This characteristic radius is large compared to the boundary layer thickness, hence fulfilling the assumption of slow radial development. It is thus legitimate to use

$$
\epsilon \equiv \frac{1}{R^{c a}} \ll 1
$$

as a small parameter in the asymptotic formulation and to introduce the slow radial coordinate

$$
\bar{R}=\epsilon r .
$$

In this multiple-scales approach, the fast $r$-scale accounts for the oscillatory behaviour of the spatially extended wavetrain, while its amplitude and local structure are slowly modulated on the $\bar{R}$-scale so as to adjust to the radial evolution of the underlying basic flow. In classical WKBJ fashion, the flow fields are expanded in powers of $\epsilon$ and written as

$$
\left\{\begin{array}{l}
\mathbf{u}(r, \theta, z, t)=\mathbf{u}(z, \phi ; \bar{R})=\mathbf{u}_{0}+\epsilon \mathbf{u}_{1}+\epsilon^{2} \mathbf{u}_{2}+\ldots \\
p(r, \theta, z, t)=p(z, \phi ; \bar{R})=p_{0}+\epsilon p_{1}+\epsilon^{2} p_{2}+\ldots
\end{array}\right.
$$



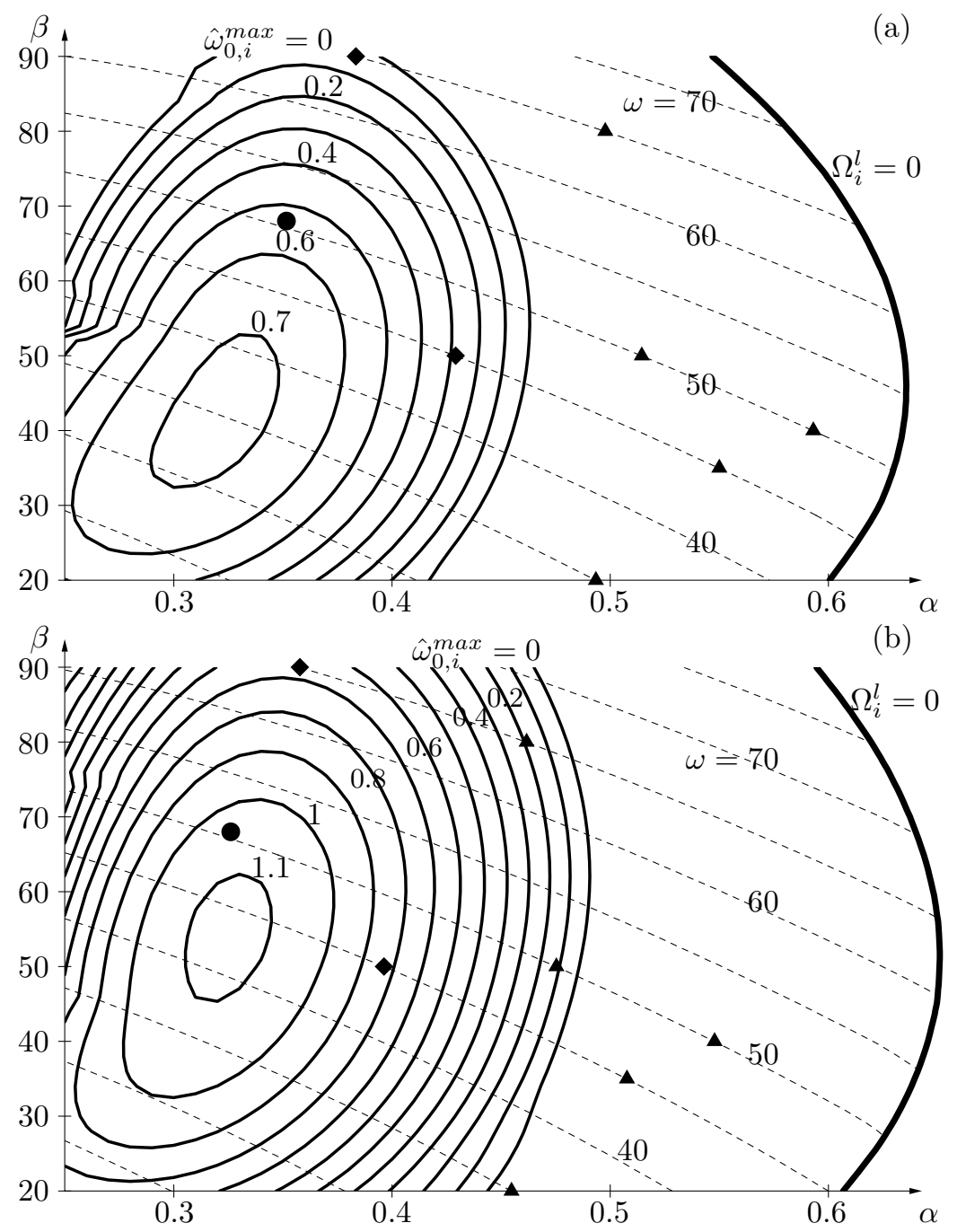

Figure 2. Isolines (thin solid curves) of nonnegative maximum secondary absolute growth rate $\hat{\omega}_{0, i}^{\max }(\alpha, \beta ; R)$ for (a) $R=500$ and (b) $R=550$. Nonlinear vortex trains exist to the left of the marginal curve $\Omega_{i}^{l}(\alpha, \beta ; R)=0$ (thick solid line) with frequencies corresponding to the dashed isolines. Symbols refer to crossflow vortices at $(\omega, \beta)=\left(\omega_{0}^{c a}, \beta^{c a}\right)$ (thick dot), $(\omega, \beta)=(65,90),(45,50)$ (diamonds), and $(\omega, \beta)=(65,80),(50,50),(50,40),(45,35),(35,20)$ (triangles).

with $2 \pi$-periodicity in the fast phase function $\phi(r, \theta, t)$ whereas the $\bar{R}$-dependence accounts for the slow radial development. For regular spatially developing global solutions, the local frequency $\omega=-\partial_{t} \phi$ and local azimuthal modenumber $\beta=\partial_{\theta} \phi$ necessarily remain constant in the entire system, while the local radial wavenumber $\alpha(\bar{R})=\partial_{r} \phi$ varies slowly with radial distance, leading to a fast phase function of 
the form

$$
\phi=\left(\frac{1}{\epsilon} \int^{\bar{R}} \alpha(\bar{\rho}) \mathrm{d} \bar{\rho}\right)+\beta \theta-\omega t .
$$

After substitution of the global solution (16) with (17) into the Navier-Stokes equations, the local governing equations are recovered at leading order in the expansion parameter $\epsilon$. At the local level, $\bar{R}$ solely acts as an external control parameter, and for each value of $\bar{R}$ the leading-order solution, among all possible waves, is the one that matches the overall frequency $\omega$ and modenumber $\beta$, as derived from the relevant dispersion relation. Note that in the previous sections, local properties were derived with the local Reynolds number $R$ as control parameter. When carrying out the present asymptotic analysis, however, it is more appropriate to rescale this parameter as $\bar{R}=\epsilon R$ and to consider the local properties as functions of the slow $\bar{R}$ instead.

Two different situations arise depending on the magnitude of the perturbation fields: in small-amplitude regions the wave pattern is described by the linearized equations while the fully nonlinear equations are needed in regions of finite amplitude vortex-trains.

Small-amplitude regions are governed by the linear dispersion relation (4). The radial wavenumber $\alpha(\bar{R})$ in the rapidly varying phase (17) is then complex, accounting for both wavelength and growth rate in the radial direction. When solving (4) for the wavenumber $\alpha$ with prescribed $\beta$ and $\omega$, two complex spatial branches $\alpha^{l \pm}(\bar{R} ; \omega, \beta)$ are obtained. The separation of these into + and - branches is dictated according to classical causality arguments $[4,2]$ and determined whether they correspond to a downstream or upstream spatial response to localized harmonic forcing. The leading-order WKBJ solution $(16,17)$ corresponding to a branch $\alpha^{l}(\bar{R} ; \omega, \beta)$ takes the form

$\mathbf{u} \sim A_{0}(\bar{R}) \mathbf{u}^{l}\left(z ; \alpha^{l}(\bar{R} ; \omega, \beta), \beta ; \bar{R}\right) \exp \mathrm{i}\left(\frac{1}{\epsilon} \int^{\bar{R}} \alpha^{l}(\bar{\rho} ; \omega, \beta) \mathrm{d} \bar{\rho}+\beta \theta-\omega t\right)$

where $\mathbf{u}^{l}$ is one of the family (3) of linear eigenfunctions and $A_{0}(\bar{R})$ is a slowly varying amplitude determined by a solvability condition at order $\epsilon$.

In contrast, the finite-amplitude régime is governed by the local nonlinear equations and the associated dispersion relation (8). Solving (8) with prescribed $\beta$ and $\omega$ yields the corresponding real wavenumber branch $\alpha^{n l}(\bar{R} ; \omega, \beta)$. In nonlinear regions, the global solution associated with $\alpha^{n l}(\bar{R} ; \omega, \beta)$ within the family (7) of saturated crossflow vortices 
has the form

$\mathbf{u} \sim \mathbf{u}^{n l}\left(z, \frac{1}{\epsilon} \int^{\bar{R}} \alpha^{n l}(\bar{\rho} ; \omega, \beta) \mathrm{d} \bar{\rho}+\beta \theta-\omega t+\Phi_{0}(\bar{R}) ; \alpha^{n l}(\bar{R} ; \omega, \beta), \beta ; \bar{R}\right)$,

where the slowly varying phase function $\Phi_{0}(\bar{R})$ obeys a solvability condition obtained at order $\epsilon$.

The stability of the spatially developing finite-amplitude vortices (19) with respect to secondary perturbations is determined by the secondary local dispersion relation (11) and in particular by the maximum secondary absolute growth rate (13) along the relevant nonlinear wavenumber branch

$$
\hat{\omega}_{0, i}^{\max }(\bar{R} ; \omega, \beta) \equiv \hat{\omega}_{0, i}^{\max }\left(\alpha^{n l}(\bar{R} ; \omega, \beta), \beta ; \bar{R}\right) .
$$

In regions where $\hat{\omega}_{0, i}^{\max }(\bar{R} ; \omega, \beta)>0$, secondary perturbations develop on top of the nonlinear WKBJ solution (19), grow at fixed radial positions and trigger transition. In contrast, while $\hat{\omega}_{0, i}^{\max }(\bar{R} ; \omega, \beta)<0$, secondary perturbations are at most convectively unstable and do not permanently affect the underlying primary wavetrain of frequency $\omega$ and modenumber $\beta$.

\section{Self-sustained behaviour}

As demonstrated in earlier investigations [32], spatially developing systems display a nonlinear self-sustained state whenever a region of absolute instability is present. This intrinsic state is the only non-trivial behaviour that would be observed in the absence of any external perturbations: a perfectly smooth disk and no residual perturbations in the surrounding fluid.

The naturally selected finite-amplitude solutions (so-called 'elephant' global modes [31]) are characterized by a stationary front located at the transition radius from local convective to absolute instability. The selection mechanism is the following: in the AU region, amplified perturbations develop and their envelope advances inwards against the radial flow. At the station of neutral absolute instability a balance between upstream perturbation growth and downstream advection is reached and perturbations pile up at that location. Nonlinearities lead to saturation of the perturation amplitude and a stationary front is formed. This front generates a downstream propagating fully nonlinear wavetrain and an upstream exponentially decaying tail. It thus connects linear and nonlinear regions, acts as a source and effectively tunes the entire system to its own frequency. The stationary front obeys a marginal 

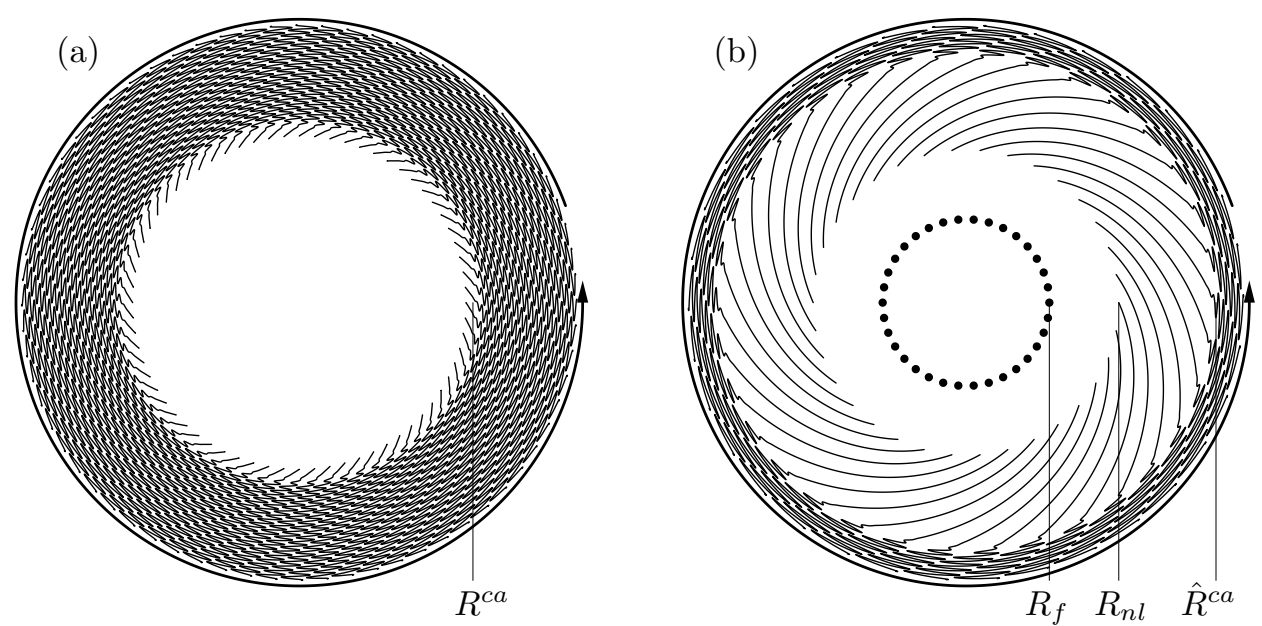

Figure 3. (a) Self-sustained flow structure. Finite-amplitude spiral vortices are triggered at $R^{c a}$, by onset of primary absolute instability, and immediately give way to turbulence, caused by secondary absolute instability. (b) Externally forced flow structure. Localised harmonic forcing applied at $R_{f}$ produces a radially amplified response. Finite-amplitude crossflow vortices develop beyond saturation radius $R^{\text {nl }}$ and break down by secondary absolute instability beyond $\hat{R}^{c a}$.

stability criterion [11], hence the global frequency of these modes equals the real absolute frequency prevailing at the front location.

In the rotating-disk flow, the absolute growth rate $\omega_{0, i}(\beta ; R)$ depends on both azimuthal modenumber and radial position, and it is for $\beta^{c a}=68$ that absolute instability first occurs: at $R^{c a} \simeq 507$ with a marginal frequency $\omega_{0}^{c a} \simeq 50.5(6)$. Hence the expected self-sustained behaviour (see sketch in figure 3a) is characterized by a front at $R^{c a}$ of frequency $\omega_{0}^{c a}$ and azimuthal modenumber $\beta^{c a}[26]$. The naturally selected flow fields can then be interpreted as the spatial response to this source, which generates the inwards exponentially decaying linear wavetrain and the outwards spiralling finite-amplitude crossflow vortices. In terms of WKBJ expansions, the inner region $R<R^{c a}$ is described by linear waves of the form (18), decaying towards the disk centre and following the complex $\alpha^{l-}\left(R ; \omega_{0}^{c a}, \beta^{c a}\right)$ radial wavenumber branch. In the outer region $R>R^{c a}$, a nonlinear wavetrain of the form (19) prevails and follows the nonlinear wavenumber branch $\alpha^{n l}\left(R ; \omega_{0}^{c a}, \beta^{c a}\right)$. Computation of $\hat{\omega}_{0, i}^{\max }\left(R ; \omega_{0}^{c a}, \beta^{c a}\right)$, the maximum secondary absolute growth rate (20) along this naturally selected nonlinear wavenumber branch (see also figure 4), reveals that the primary saturated waves initiated by the front at $R^{c a}$ are already absolutely unstable with respect to secondary perturbations [26]. 
In view of these results, the intrinsic behaviour of the rotating-disk boundary layer (figure 3a) may be explained in the following way. The self-sustained finite-amplitude fluctuations are produced at the inner boundary $R^{c a}$ of the absolutely unstable domain. This frontier for onset of primary absolute instability acts as a source and generates outwards spiralling saturated crossflow vortices, governed locally by the associated nonlinear dispersion relation. Due to secondary absolute instability, this naturally selected primary structure is dynamically unstable and immediately gives way to a disordred state.

\section{Externally forced behaviour}

The rotating disk boundary layer is convectively unstable over the radial interval $R^{s c} \simeq 284<R<R^{c a} \simeq 507$ and can thus also act as an amplifier of external perturbations, such as roughness elements on the disk surface or fluctuations in the external flow. In order to characterize the response of the boundary layer to external perturbations, this section addresses the signalling problem: the spatial response to radially localized harmonic forcing applied in the at most $\mathrm{CU}$ domain (see sketch in figure 3b).

Consider a radially localized forcing at $R_{f}$ with frequency $\omega_{f}$, azimuthal modenumber $\beta_{f}$ and small amplitude $A_{f}$. In the vicinity of the forcing location, the magnitude of the response is of the same order as the forcing amplitude and thus governed by linear dynamics, provided that $A_{f} \ll 1$. Near $R_{f}$ the spatial response then follows a linear WKBJ expansion of the form (18) where the complex local radial wavenumber branches $\alpha^{l \pm}\left(R ; \omega_{f}, \beta_{f}\right)$ are obtained by solving (4) with $\omega=\omega_{f}$ and $\beta=\beta_{f}$ : the $\alpha^{l+}$-branch pertains to the outwards $R>R_{f}$ side of the forcing and the $\alpha^{l-}$-branch to the inwards $R<R_{f}$ side.

In stable or CU regions, the upstream spatial response decays for all frequencies and modenumbers, thus $\alpha_{i}^{l-}\left(R ; \omega_{f}, \beta_{f}\right)<0$ for all $R<R_{f}$. The linear WKBJ approximation (18) which is exponentially decaying towards the disk axis with local wavenumber $\alpha^{l-}\left(R ; \omega_{f}, \beta_{f}\right)$ then applies to the entire region upstream of the forcing location.

For forcing applied at $R_{f}$ in the $\mathrm{CU}$ domain, there exists however a range of frequencies and modenumbers yielding downstream growth, i.e., with $\alpha_{i}^{l+}\left(R_{f} ; \omega_{f}, \beta_{f}\right)<0$. At leading order, the order of magnitude, $\psi_{\text {ext }}$, of the externally forced linear spatial response (18) for $R>R_{f}$ is

$$
\psi_{e x t} \sim A_{f} \exp \int_{R_{f}}^{R}-\alpha_{i}^{l+}\left(\rho ; \omega_{f}, \beta_{f}\right) \mathrm{d} \rho
$$

cdisk.tex; 29/8/2006; ; p.13 
and hence grows exponentially radially outwards. The nonlinear saturation station $R^{n l}$ where the spatial response takes $\mathcal{O}(1)$ values is determined by the condition

$$
\int_{R_{f}}^{R^{n l}}-\alpha_{i}^{l+}\left(\rho ; \omega_{f}, \beta_{f}\right) \mathrm{d} \rho=-\log A_{f} .
$$

At $R^{n l}$, nonlinear saturation prevents further growth and leads to a nonlinear wavetrain, again with frequency and modenumber determined by the forcing. Beyond $R^{n l}$, the spatial response thus consists of a finite-amplitude saturated wavetrain of the form (19), uniquely determined by the forcing parameters $\omega_{f}$ and $\beta_{f}$, and whose local radial wavenumber follows the nonlinear branch $\alpha^{n l}\left(R ; \omega_{f}, \beta_{f}\right)$.

The long-term stability of these primary nonlinear spiral vortices is dictated by $\hat{\omega}_{0, i}^{\max }\left(R ; \omega_{f}, \beta_{f}\right)$ the maximum secondary absolute growth rate (20) following along the nonlinear wavenumber branch $\alpha^{n l}\left(R ; \omega_{f}, \beta_{f}\right)$ (see figure 4$)$. Denote by $\hat{R}^{c a}\left(\omega_{f}, \beta_{f}\right)$ the radius corresponding to transition from secondary convective to absolute instability, i.e., defined by

$$
\hat{\omega}_{0, i}^{\max }\left(R ; \omega_{f}, \beta_{f}\right)=0 \quad \text { for } \quad R=\hat{R}^{c a}\left(\omega_{f}, \beta_{f}\right) .
$$

For $R<\hat{R}^{c a}\left(\omega_{f}, \beta_{f}\right)$, a secondary perturbation is at most convectively unstable $\left(\hat{\omega}_{0, i}^{\max }\left(R ; \omega_{f}, \beta_{f}\right)<0\right)$ and thus does not succeed in permanently affecting the primary crossflow vortices. For $R>\hat{R}^{c a}\left(\omega_{f}, \beta_{f}\right)$, however, the AU finite-amplitude crossflow vortices $\left(\hat{\omega}_{0, i}^{\max }\left(R ; \omega_{f}, \beta_{f}\right)>\right.$ $0)$ give way to a disordered state.

It should be noted that, for given $\omega_{f}$ and $\beta_{f}$, the saturation location $R^{n l}$ depends on both forcing amplitude $A_{f}$ and radius $R_{f}$, whereas the nonlinear wavetrain (19) prevailing beyond $R^{\text {nl }}$ does not. Thus the radius $\hat{R}^{c a}\left(\omega_{f}, \beta_{f}\right)$ for onset of secondary absolute instability is uniquely determined by the forcing parameters $\omega_{f}$ and $\beta_{f}$, while that, $R^{n l}$, for primary nonlinearity further depends on the parameters $A_{f}$ and $R_{f}$.

The character of the forced spatial response depends on the relative positions of $\hat{R}^{c a}$ and $R^{n l}$. In situations where $R^{n l}<\hat{R}^{c a}$ (sketched in figure $3 \mathrm{~b}$ ), the linear spatial response (18) grows from $R_{f}$ to $R^{n l}$, followed by nonlinear periodic crossflow vortices (19) in the domain $R^{n l}<R<\hat{R}^{c a}$. Secondary absolute instability occurs at $\hat{R}^{c a}$, leading to a disordered state in $R>\hat{R}^{c a}$. An increase/decrease of the forcing amplitude $A_{f}$ results in earlier/later onset of nonlinearity $\left(R^{n l}\right)$ but does not modify the secondary stability properties nor transition at $\hat{R}^{c a}\left(\omega_{f}, \beta_{f}\right)$.

With very low forcing amplitudes, onset of nonlinearity may be delayed beyond $\hat{R}^{c a}$, i.e., $R^{n l}>\hat{R}^{c a}$. Near $R^{n l}$, nonlinear saturation then 
leads to a wavetrain which is already $\mathrm{AU}$ with respect to secondary perturbations $\left(\hat{\omega}_{0, i}^{\max }\left(R^{n l} ; \omega_{f}, \beta_{f}\right)>0\right)$. Thus the nonlinear periodic régime (19) is bypassed and a disordered state covers the entire region beyond $R^{n l}$. Since the transition radius then directly depends on $R^{n l}$, an increase/decrease of the forcing amplitude $A_{f}$ then brings about earlier/later transition. Due to the exponential growth of the response, a situation where $R^{\text {nl }}>\hat{R}^{c a}$ generally occurs, however, only for extremely small forcing amplitudes.

\section{Open-loop control}

The above results show that the rotating-disk boundary layer displays all the features required for successful implementation of the openloop control method previously developed for a one-dimensional model problem [27]. In this strategy, localized periodic forcing is applied in the $\mathrm{CU}$ region so as to replace the naturally selected nonlinear global structure by the spatial response to external forcing. The aim is not to suppress the primary vortices but to tune them to an externally imposed frequency and modenumber and thereby delay onset of secondary absolute instability and transition.

Assuming that the boundary layer displays the self-sustained behaviour described in Sect. 6, the AU domain $R>R^{c a} \simeq 507$ is covered by finite-amplitude fluctuations initiated at $R^{c a}$, while a linear WKBJ approximation (18) of frequency $\omega_{0}^{c a}$ and modenumber $\beta^{c a}$ describes the inner range $R<R^{c a}$. The resulting linear wavetrain decays exponentially towards the disk centre and, to leading order, the order of magnitude, $\psi_{\text {int }}$, of its amplitude varies with radial distance $R$ as

$$
\log \psi_{i n t} \sim \int_{R}^{R^{c a}} \alpha_{i}^{l-}\left(\rho ; \omega_{0}^{c a}, \beta^{c a}\right) \mathrm{d} \rho .
$$

Suppose that a radially localised external forcing of frequency $\omega_{f}$ and modenumber $\beta_{f}$ is applied to the above flow structure at $R_{f}$ in the CU region, i.e., $R^{s c}<R_{f}<R^{c a}$. For small forcing amplitude $A_{f}$, the order of magnitude (21) of the linear spatial response for $R>R_{f}$ is given by

$$
\log \psi_{e x t} \sim a_{f}-\int_{R_{f}}^{R} \alpha_{i}^{l+}\left(\rho ; \omega_{f}, \beta_{f}\right) \mathrm{d} \rho,
$$

where $a_{f}=\log A_{f}$. In the neighbourhood of $R_{f}$, both the spatial response and the self-sustained global mode are governed by linear dynamics and the resulting flow is a superposition of both fields. This linear régime prevails through the region extending from $R_{f}$ outwards 
until either $\psi_{\text {ext }}$ or $\psi_{\text {int }}$ reaches finite levels. Nonlinearity of the selfsustained field appears at $R^{c a}$, whereas the forced response achieves $\mathcal{O}(1)$ amplitude at the saturation radius $R^{n l}$, defined by (22) and which depends on the forcing parameters. The nature of the nonlinear dynamics taking over from the linear régime thus crucially depends on the relative positions of $R^{c a}$ and $R^{n l}$.

For given $\omega_{f}, \beta_{f}$ and $R_{f}$, there exists a critical forcing amplitude $A_{c} \equiv \mathrm{e}^{a_{c}}$, defined by

$$
a_{c}=\int_{R_{f}}^{R^{c a}} \alpha_{i}^{l+}\left(\rho ; \omega_{f}, \beta_{f}\right) \mathrm{d} \rho
$$

for which the nonlinear saturation radius $R^{n l}$ of the externally forced response coincides with the onset radius $R^{c a}$ of intrinsic nonlinearities. For stronger forcing levels $A_{f}>A_{c}$ (resp. weaker levels $A_{f}<A_{c}$ ), the saturation radius moves upstream $R^{n l}<R^{c a}$ (resp. downstream $\left.R^{n l}>R^{c a}\right)$.

The open-loop control strategy [27] to be applied here for the rotatingdisk boundary layer is based on the following results. For weak forcing levels $A_{f}<A_{c}$, the spatial response does not achieve $\mathcal{O}(1)$ amplitudes at radius $R^{c a}$ and is thus unable to perturb the nonlinear self-sustained state selected by the front at $R^{c a}$ and triggering finite-amplitude fluctuations for $R>R^{c a}$. However, for higher forcing levels $A_{f}>A_{c}$, the spatial response reaches nonlinear saturation upstream of the front, i.e., $R^{\text {nl }}<R^{c a}$, and the naturally selected behaviour is then suppressed and replaced by the forced spatial response throughout the flow.

This behaviour may be interpreted as the result of two competing sources of different periodicities at different locations: the self-sustained $\left(\omega_{0}^{c a}, \beta^{c a}\right)$-front at $R^{c a}$ (responsible for the intrinsic nonlinear structure) and the external $\left(\omega_{f}, \beta_{f}\right)$-forcing at $R_{f}$. In the absence of external forcing, the front at $R^{c a}$ acts as a keystone upon which the global structure is based. When forcing is applied at $R_{f}$, the intrinsic wavemaker at $R^{c a}$ survives only if its upstream decaying tail experiences an unperturbed medium. As soon as the front is overwhelmed by incoming finite-amplitude perturbations, the source of the global mode is suppressed and hence so is the entire self-sustained structure. The underlying (primary) AU region then plays no rôle in the dynamics, since it is effectively masked by an externally imposed nonlinear wavetrain.

Without external input, the boundary layer displays transition to turbulence near $R^{c a} \simeq 507$ (figure $3 \mathrm{a}$ ). With open-loop control by external forcing of periodicity $\omega_{f}$ and $\beta_{f}$, transition occurs instead near $\hat{R}^{c a}\left(\omega_{f}, \beta_{f}\right)$, where the externally forced nonlinear crossflow vortices become AU with respect to secondary perturbations (figure $3 \mathrm{~b}$ ). The

cdisk.tex; 29/8/2006; ; p.16 
goal of delaying transition can then be achieved if the two conditions

$$
R^{n l}<R^{c a} \text { and } \hat{R}^{c a}>R^{c a}
$$

are both fulfilled. Identification of efficient control parameters thus requires a systematic investigation of primary and secondary instability characteristics.

The condition $R^{n l}<R^{c a}$ is necessary for control of the primary wavetrain to be effective: the forced spatial response needs to reach nonlinear levels and to saturate upstream of $R^{c a}$ in order to supersede the self-selected dynamics. The second condition, $\hat{R}^{c a}>R^{c a}$, then guarantees that onset of secondary absolute instability, and thus of transition, is postponed to beyond $R^{c a}$. Thus the forcing parameters $\omega_{f}$ and $\beta_{f}$ must be chosen so that, near $R^{c a}$, the resulting nonlinear crossflow vortices have negative secondary absolute growth rate.

Suitable control parameters may be derived from figure 2(a) which shows the $(\alpha, \beta)$-plane for $R=500$, slightly upstream of $R^{c a}$. Saturated travelling waves exist to the left of the marginal boundary $\Omega_{i}^{l}(\alpha, \beta)=0$ (thick solid curve) and their nonlinear frequencies $\Omega^{n l}(\alpha, \beta)$ are indicated by dashed curves. Among these nonlinear waves, those associated with secondary absolute instability $\left(\hat{\omega}_{0, i}^{\max } \geq 0\right.$, indicated by thin solid isocontours) must be avoided. As a result, the two control conditions (27) may be met for frequencies $\omega_{f}$ and modenumbers $\beta_{f}$ associated with nonlinear vortices located between the curves $\Omega_{i}^{l}=0$ and $\hat{\omega}_{0, i}^{\max }=0$. In figure $2(\mathrm{a})$, selected forcing parameters are indicated by symbols: transition can be delayed for $\left(\omega_{f}, \beta_{f}\right)=(65,80),(50,50)$, $(50,40),(45,35)$ or $(35,20)$ (triangles), but not for $\left(\omega_{f}, \beta_{f}\right)=(65,90)$, $(45,50)$ (diamonds), nor of course for the self-sustained $\left(\omega_{0}^{c a}, \beta^{c a}\right)$ (thick dot).

With external forcing, the new transition radius $\hat{R}^{c a}\left(\omega_{f}, \beta_{f}\right)$ is determined by the zero crossing of the maximum secondary absolute growth rate $\hat{\omega}_{0, i}^{\max }\left(R ; \omega_{f}, \beta_{f}\right)$. Figure $4(\mathrm{a})$ shows the radial evolution of $\hat{\omega}_{0, i}^{\max }$ for different values of $\omega_{f}$ and $\beta_{f}$; the associated nonlinear wavenumber branches $\alpha^{n l}\left(R ; \omega_{f}, \beta_{f}\right)$ are given in figure $4(\mathrm{~b})$. The values of $\hat{\omega}_{0, i}^{\max }$ for $R=500$ and $R=550$, corresponding to the forcing parameters used in figure 4 , are shown by symbols in figures $2(\mathrm{a})$ and (b) respectively.

It is seen that the naturally selected vortices, for $\omega_{0}^{c a}$ and $\beta^{c a}$ (corresponding to the solid dot in figure 2), are among the most unstable primary nonlinear waves. The associated $\hat{\omega}_{0, i}^{\max }$ and $\alpha^{n l}$ curves are given in figure 4 for $400<R<600$, but note that the values for $R<R^{c a}$ are irrelevant to the self-sustained global mode, since it has finite amplitude only for $R>R^{c a}$.

When the intrinsic dynamics at $\left(\omega_{0}^{c a}, \beta^{c a}\right)$ is replaced by nonlinear waves with $\left(\omega_{f}, \beta_{f}\right)=(65,90)$ or $(45,50)$ (diamonds in figure 2$)$, sec- 

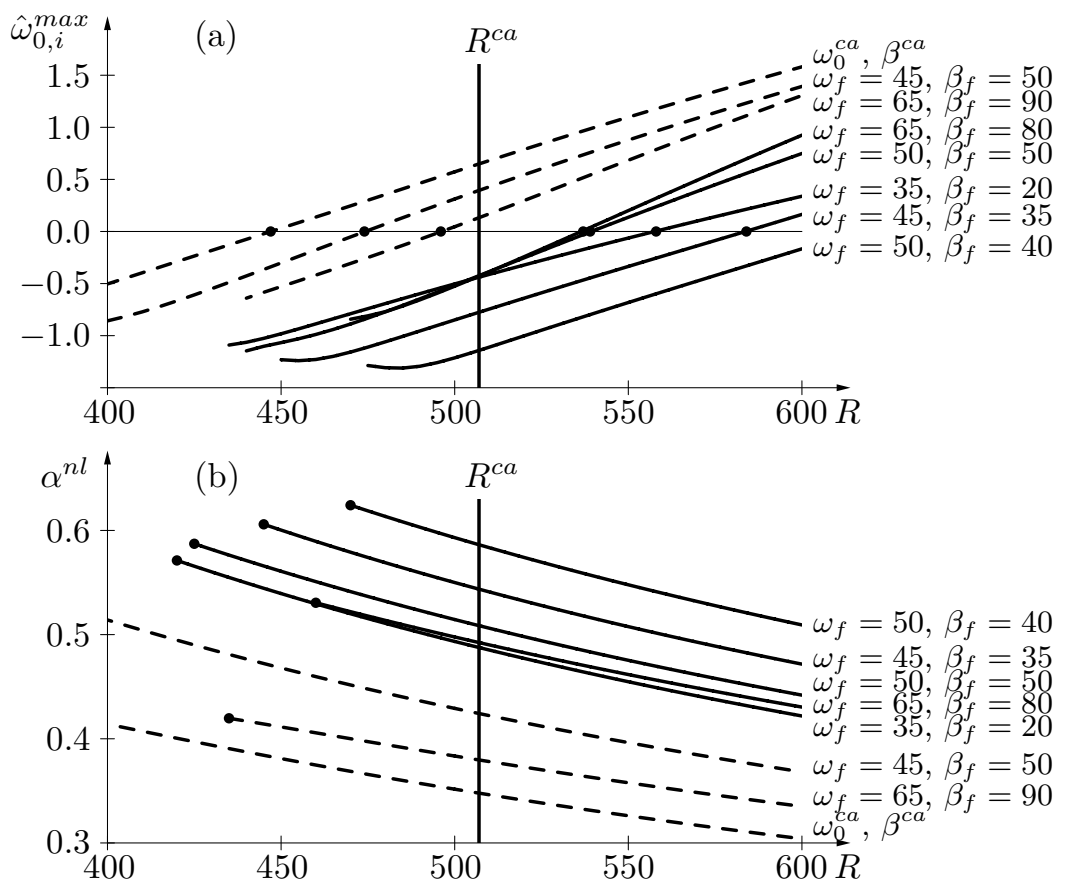

Figure 4. (a) Maximum secondary absolute growth rate $\hat{\omega}_{0, i}^{\max }\left(R ; \omega_{f}, \beta_{f}\right)$ and (b) nonlinear spatial branches $\alpha^{n l}\left(R ; \omega_{f}, \beta_{f}\right)$ for different values of $\omega_{f}$ and $\beta_{f}$. The zero-crossings of $\hat{\omega}_{0, i}^{\max }$ in (a) define the corresponding $\hat{R}^{c a}\left(\omega_{f}, \beta_{f}\right)$ and are marked by dots. The $\alpha^{n l}$ branches in (b) terminate at low $R$ when the marginal $R^{m}\left(\omega_{f}, \beta_{f}\right)$ is reached. Solid lines in (a) and (b) correspond to forcing parameters which delay onset of secondary absolute instability to beyond $R^{c a}$.

ondary instability is reduced and the $\hat{\omega}_{0, i}^{\max }$ curves in figure $4(\mathrm{a})$ are lowered. For these forcing parameters, however, the radius $R^{c a}$ remains within the secondarily AU region $\hat{\omega}_{0, i}^{\max }>0$. In consequence, rather than reducing the size of the turbulent domain, external harmonic forcing actually promotes earlier transition because the secondary perturbations propagate inwards below $R^{c a}$ down to the corresponding marginal radius $\hat{R}^{c a}\left(\omega_{f}, \beta_{f}\right)<R^{c a}$ (indicated by small dots in figure $\left.4 \mathrm{a}\right)$.

External forcing of nonlinear waves between the marginal $\Omega_{i}^{l}=0$ and $\hat{\omega}_{0, i}^{\max }=0$ curves in figure 2(a) sufficiently weakens secondary instability that the critical radius $\hat{R}^{c a}$ for onset of secondary absolute growth is located beyond $R^{c a}$, fulfilling the second condition (27). Values corresponding to $\left(\omega_{f}, \beta_{f}\right)=(65,80),(50,50),(50,40),(45,35)$ and $(35,20)$ are indicated by triangles in figure 2 , and the corresponding $\hat{\omega}_{0, i}^{\max }$ and $\alpha^{n l}$ branches are represented by solid lines in figure 4 . With $\omega_{f}=50$ and $\beta_{f}=40$ (lowest curve in figure $4 \mathrm{a}$ ), onset of secondary absolute 
instability is postponed to beyond $\hat{R}^{c a}>600$. Hence it is possible to delay the turbulent régime by approximately 100 boundary layer units from $R^{c a}$ to $\hat{R}^{c a}$.

Due to the condition $R^{n l}<R^{c a}$, it seems unlikely that forcing parameters exist that would delay transition much further. Indeed, the nonlinear solution branches are governed by the local nonlinear dispersion relation (8) and terminate at low $R$ when the marginal radius associated with $\omega_{f}$ and $\beta_{f}$, denoted as $R^{m}\left(\omega_{f}, \beta_{f}\right)$ and indicated by dots in figure $4(\mathrm{~b})$, is reached. Thus for given $\omega_{f}$ and $\beta_{f}$, saturation of the spatial response may only occur for $R^{n l}>R^{m}\left(\omega_{f}, \beta_{f}\right)$, and the range of possible control parameters is therefore limited by the condition

$$
R^{m}\left(\omega_{f}, \beta_{f}\right)<R^{c a} .
$$

As can be seen from figure 4 , settings of $\omega_{f}$ and $\beta_{f}$ that yield high values of $\hat{R}^{c a}\left(\omega_{f}, \beta_{f}\right)$ also push $R^{m}\left(\omega_{f}, \beta_{f}\right)$ outwards.

The spatial response at $\omega_{f}$ and $\beta_{f}$ is radially exponentially amplified over the interval $R^{m}\left(\omega_{f}, \beta_{f}\right)<R<R^{c a}$, and the largest amplification is obtained when forcing is applied at $R_{f}=R^{m}\left(\omega_{f}, \beta_{f}\right)$. Due to the exponential growth of the forced response, only small forcing amplitudes (26) are generally necessary to reach a nonlinear state at $R^{n l}<R^{c a}$. However, when $R^{m}$ is too close to $R^{c a}$, the radial amplification of the spatial response is only moderate and more substantial forcing amplitudes are required.

As a result, the values of $\omega_{f}=50$ and $\beta_{f}=40$ are deemed to be very close to the optimal forcing parameters for delaying transition by the present open-loop control method.

\section{Discussion}

For the three-dimensional boundary layer produced by a rotating disk, the scenario leading from the unperturbed boundary layer to the turbulent state takes place in two steps and involves both primary and secondary instabilities: primary nonlinear waves are the prerequisite for a possible development of secondary absolute instability leading to transition. Since the secondary disturbances feed on the primary vortices, the turbulent régime prevailing at large radial distances may propagate inwards until either the nonlinear waves cease to exist $(R=$ $\left.R^{n l}\right)$ or until their secondary absolute growth rate changes $\operatorname{sign}(R=$ $\left.\hat{R}^{c a}\right)$.

The intrinsic dynamics, observed without external perturbations, display a sudden transition from basic to turbulent states, where primary and secondary instabilities simultaneously take place. At the ra-

cdisk.tex; 29/8/2006; ; p.19 
dius $R^{c a} \simeq 507$ of transition from primary linear convective to absolute instability, a stationary front of frequency $\omega_{0}^{c a} \simeq 50.5$ and azimuthal modenumber $\beta^{c a}=68$ generates outward spiralling nonlinear crossflow vortices. These finite-amplitude waves are already AU with respect to secondary perturbations. Hence a disordered state covers the whole region $R>R^{c a}$, while the unperturbed boundary layer prevails for $R<R^{c a}$. In this situation, the transition location is dictated by onset of primary nonlinear waves, which in turn corresponds to onset of primary absolute instability $\left(R^{n l}=R^{c a}\right)$.

When harmonic forcing is applied at $R_{f}$ in the CU region with frequency $\omega_{f}$ and modenumber $\beta_{f}$ in the unstable range, the linear response exponentially grows with radial distance and reaches finiteamplitude at radius $R^{n l}>R_{f}$. The associated nonlinear vortices display secondary absolute instability for $R>\hat{R}^{c a}$. When forcing parameters are chosen so that $R^{n l}<\hat{R}^{c a}$, the spatial response displays three successive régimes downstream of $R_{f}$ : linear growth over $R_{f}<$ $R<R^{n l}$, nonlinear crossflow vortices over $R^{n l}<R<\hat{R}^{c a}$ and a turbulent state for $R>\hat{R}^{c a}$. In this situation, transition is due to onset of secondary absolute instability at $\hat{R}^{c a}$, and this radius is uniquely determined by $\omega_{f}$ and $\beta_{f}$.

The aim of the open-loop control strategy is to delay onset of secondary AU perturbations, and thus transition, from $R^{c a}$ to larger radii by a controlled modification of the primary nonlinear state. This technique consists in replacing the naturally selected flow state by the spatial response to carefully chosen harmonic forcing. Transition is effectively postponed for control parameters such that $R^{n l}<R^{c a}<$ $\hat{R}^{c a}$ : by enhancing primary instability, onset of secondary instability may be delayed. Thus the natural dynamics, where primary and secondary instabilities occur simultaneously at $R^{c a}$, is replaced by an externally forced flow structure whose primary nonlinearities appear earlier (at $R^{n l}<R^{c a}$ ) but whose secondary perturbations develop only later (at $\left.\hat{R}^{c a}>R^{c a}\right)$. In other words, the linear $\left(\omega_{f}, \beta_{f}\right)$-waves must be sufficiently unstable to reach nonlinear saturation before $R^{c a}$ and at the same time not too unstable so that the resulting finite-amplitude waves display secondary absolute instability only after $R^{c a}$. Best control (large $\hat{R}^{c a}$ ) is thus obtained by applying weakly unstable forcing: transition may be delayed by approximately 100 boundary layer units beyond $R^{c a}$ when using $\omega_{f}=50$ and $\beta_{f}=40$. However, optimizing for large $\hat{R}^{c a}$ requires a very precise tuning of the forcing parameters and is expected to be difficult to implement experimentally. 


\section{References}

1. Bender, C. M. and S. A. Orszag: 1978, Advanced mathematical methods for scientists and engineers. New York: McGraw-Hill.

2. Bers, A.: 1983, 'Space-time evolution of plasma instabilities - absolute and convective'. In: M. Rosenbluth and R. Sagdeev (eds.): Handbook of plasma physics. Amsterdam: North-Holland, pp. 451-517.

3. Brevdo, L. and T. J. Bridges: 1996, 'Absolute and convective instabilities of spatially periodic flows'. Phil. Trans. R. Soc. Lond. A 354, 1027-1064.

4. Briggs, R. J.: 1964, Electron-stream interaction with plasmas. Cambridge, Mass.: M.I.T. Press.

5. Chomaz, J.-M., P. Huerre, and L. G. Redekopp: 1991, 'A frequency selection criterion in spatially developing flows'. Stud. Appl. Math. 84, 119-144.

6. Couairon, A. and J.-M. Chomaz: 1996, 'Global instabilities in fully nonlinear systems'. Phys. Rev. Lett. 77, 4015-4018.

7. Couairon, A. and J.-M. Chomaz: 1997, 'Absolute and convective instabilities, front velocities and global modes in nonlinear systems'. Physica D 108, 236276.

8. Couairon, A. and J.-M. Chomaz: 1999, 'Fully nonlinear global modes in slowly varying flows'. Phys. Fluids 11, 3688-3703.

9. Davies, C. and P. W. Carpenter: 2003, 'Global behaviour corresponding to the absolute instability of the rotating-disk boundary layer'. J. Fluid Mech. 486, 287-329.

10. Davies, C., C. Thomas, and P. W. Carpenter: 2006, 'Global stability of the rotating-disc boundary layer'. J. Eng. Mech.

11. Dee, G. and J. S. Langer: 1983, 'Propagating pattern selection'. Phys. Rev. Lett. 50, 383-386.

12. Garrett, S. J.: 2002, 'The stability and transition of the boundary layer on rotating bodies'. Ph.D. thesis, University of Cambridge, Cambridge.

13. Gregory, N., J. T. Stuart, and W. S. Walker: 1955, 'On the stability of threedimensional boundary layers with application to the flow due to a rotating disk'. Phil. Trans. R. Soc. Lond. A 248, 155-199.

14. Herbert, T.: 1988, 'Secondary instability of boundary layers'. Ann. Rev. Fluid Mech. 20, 487-526.

15. Huerre, P.: 2000, 'Open shear flow instabilities'. In: G. K. Batchelor, H. K. Moffatt, and M. G. Worster (eds.): Perspectives in Fluid Dynamics. Cambridge: Cambridge University Press, pp. 159-229.

16. Huerre, P. and P. A. Monkewitz: 1990, 'Local and global instabilities in spatially developing flows'. Ann. Rev. Fluid Mech. 22, 473-537.

17. Jarre, S., P. Le Gal, and M. P. Chauve: 1996, 'Experimental study of rotating disk flow instability. II. Forced flow'. Phys. Fluids 8, 2985-2994.

18. Kohama, Y.: 1984, 'Study on boundary layer transition of a rotating disk'. Acta Mechanica 50, 193-199.

19. Lingwood, R. J.: 1995, 'Absolute instability of the boundary layer on a rotating disk'. J. Fluid Mech. 299, 17-33.

20. Lingwood, R. J.: 1996, 'An experimental study of absolute instability of the rotating-disk boundary-layer flow'. J. Fluid Mech. 314, 373-405.

21. Lingwood, R. J.: 1997, 'Absolute instability of the Ekman layer and related rotating flows'. J. Fluid Mech. 331, 405-428.

22. Monkewitz, P. A., P. Huerre, and J.-M. Chomaz: 1993, 'Global linear stability analysis of weakly non-parallel shear flows'. J. Fluid Mech. 251, 1-20. 
23. Othman, H. and T. Corke: 2006, 'Experimental investigation of absolute instability of a rotating-disk boundary layer'. J. Fluid Mech. in press.

24. Peake, N. private communication.

25. Pier, B.: 2002, 'Fully nonlinear waves and transition in the boundary layer over a rotating disk'. In: I. P. Castro and P. E. Hancock (eds.): Advances in Turbulence IX, Proceedings of the 9th European Turbulence Conference. Barcelona, pp. 13-16.

26. Pier, B.: 2003a, 'Finite-amplitude crossflow vortices, secondary instability and transition in the rotating-disk boundary layer'. J. Fluid Mech. 487, 315-343.

27. Pier, B.: 2003b, 'Open-loop control of absolutely unstable domains'. Proc. R. Soc. Lond. A 459, 1105-1115.

28. Pier, B.: 2004, 'Open-loop control and delay of transition in the rotating-disk boundary layer'. In: H. I. Andersson and P.-A. Krogstad (eds.): Advances in Turbulence X, Proceedings of the 10th European Turbulence Conference. Barcelona, pp. $775-778$.

29. Pier, B. and P. Huerre: 1996, 'Fully nonlinear global modes in spatially developing media'. Physica D 97, 206-222.

30. Pier, B. and P. Huerre: 2001a, 'Nonlinear self-sustained structures and fronts in spatially developing wake flows'. J. Fluid Mech. 435, 145-174.

31. Pier, B. and P. Huerre: 2001b, 'Nonlinear synchronization in open flows'. J. Fluids Struct. 15, 471-480.

32. Pier, B., P. Huerre, and J.-M. Chomaz: 2001, 'Bifurcation to fully nonlinear synchronized structures in slowly varying media'. Physica D 148, 49-96.

33. Pier, B., P. Huerre, J.-M. Chomaz, and A. Couairon: 1998, 'Steep nonlinear global modes in spatially developing media'. Phys. Fluids 10, 2433-2435.

34. Reed, H. L. and W. S. Saric: 1989, 'Stability of three-dimensional boundary layers'. Ann. Rev. Fluid Mech. 21, 235-284.

35. Saric, W. S., H. L. Reed, and E. B. White: 2003, 'Stability and transition of three-dimensional boundary layers'. Annu. Rev. Fluid Mech. 35, 413-440.

36. Schmid, P. J. and D. S. Henningson: 2001, Stability and transition in shear flows, Applied Mathematical Sciences. New York: Springer.

37. von Kármán, T.: 1921, 'Über laminare und turbulente Reibung'. Z. Angew. Math. Mech. 1, 232-252.

38. Wilkinson, S. P. and M. R. Malik: 1985, 'Stability experiments in the flow over a rotating disk'. AIAA J. 23, 588-595. 